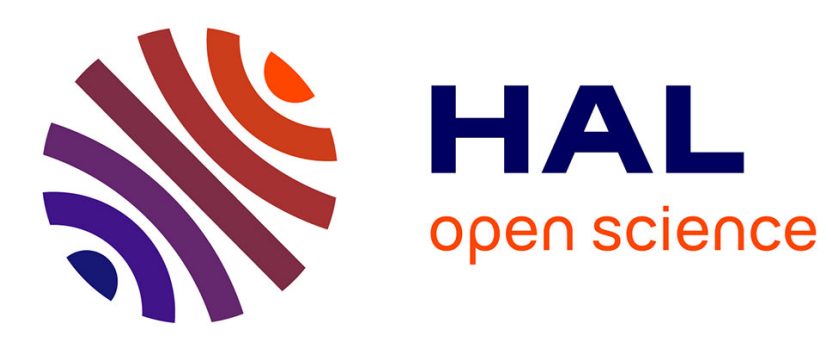

\title{
Existence of solutions to a phase-field model for the isothermal solidification process of a binary alloy
}

\author{
Jacques Rappaz, Jean-Francois Scheid
}

\section{To cite this version:}

Jacques Rappaz, Jean-Francois Scheid. Existence of solutions to a phase-field model for the isothermal solidification process of a binary alloy. Mathematical Methods in the Applied Sciences, 2000, 23 (6), pp.491-513. 10.1002/(SICI)1099-1476(200004)23:63.0.CO;2-4 . hal-03116846

\section{HAL Id: hal-03116846 \\ https://hal.science/hal-03116846}

Submitted on 20 Jan 2021

HAL is a multi-disciplinary open access archive for the deposit and dissemination of scientific research documents, whether they are published or not. The documents may come from teaching and research institutions in France or abroad, or from public or private research centers.
L'archive ouverte pluridisciplinaire HAL, est destinée au dépôt et à la diffusion de documents scientifiques de niveau recherche, publiés ou non, émanant des établissements d'enseignement et de recherche français ou étrangers, des laboratoires publics ou privés. 


\title{
Existence of solutions to a phase-field model for the isothermal solidification process of a binary alloy *
}

\author{
J. Rappaz ${ }^{1}$ J.F. Scheid ${ }^{2}$
}

\begin{abstract}
We investigate the well-posedness of a phase-field model for the isothermal solidification of a binary alloy due to Warren-Boettinger [12]. Existence of weak solution as well as regularity and uniqueness results are established under Lipschitz and boundeness assumptions for the nonlinearities. A maximum principle holds that guarantees the existence of a solution under physical assumptions on the nonlinearities.
\end{abstract}

MOS subject classification : 82C26, 35K55, 35B65, 35B50.

\section{Introduction.}

In this paper we study the well-posedness of a solutal phase-field model for the solidification of a binary alloy at a constant temperature. Such a model was derived by Warren-Boettinger [12]. It involves the relative concentration $c$ and an order parameter $\phi$ which accounts for the solidification state of the alloy by being equal to 0 if the system is in a solid phase and equal to 1 if it is in a liquid phase. The time evolution of $c$ and $\phi$ is governed by the following equations :

$$
(P) \begin{cases}\frac{\partial \phi}{\partial t}=\varepsilon^{2} \Delta \phi+F_{1}(\phi)+c F_{2}(\phi) & \text { in } \Omega \times(0,+\infty), \\ \frac{\partial c}{\partial t}=\operatorname{div}\left(D_{1}(\phi) \nabla c+D_{2}(c, \phi) \nabla \phi\right) & \text { in } \Omega \times(0,+\infty), \\ \frac{\partial \phi}{\partial n}=\frac{\partial c}{\partial n}=0 & \text { on } \partial \Omega \times(0,+\infty), \\ \phi(0)=\phi_{0}, \quad c(0)=c_{0} & \text { in } \Omega,\end{cases}
$$

where $\Omega$ is an open subset of $\mathbb{R}^{d}$ with $1 \leq d \leq 3$ and with boundary $\partial \Omega, n$ is the unit normal to $\partial \Omega$ and $\varepsilon>0$ is a given positive constant.

The functions $F_{1}, F_{2}, D_{1}$ and $D_{2}$ appearing in $(P)$ have the following properties:

- $\quad F_{1}$ and $F_{2}$ are regular functions such that $F_{i}(0)=F_{i}(1)=0$ for $i=1,2$.

- $D_{1}$ is a positive and regular function bounded above and below by two positive constants.

- $D_{2}$ is a regular function such that $D_{2}(c,)=$.0 for $c=0$ and $c=1$.

Moreover, initial physical data $c_{0}$ and $\phi_{0}$ are given and have to stay between 0 and 1 . The solutions $c$ and $\phi$ of Problem $(P)$ must be found with the same property.

Phase-field models have been principally used to describe phase transitions of pure materials due to thermal effects and they lead to nonlinear parabolic systems for the phase-field and the temperature. However, the nonlinearities are then different from those of problem $(P)$. Such thermal models have been well studied and we refer for instance to [2], [3], [1] and [8] where existence and uniqueness results are investigated.

\footnotetext{
*supported by the Swiss National Foundation.

${ }^{1}$ Département de mathématiques, Ecole Polytechnique Fédérale de Lausanne, 1015 Lausanne, Switzerland; email : Jacques.Rappaz@epfl.ch

${ }^{2}$ Département de mathématiques, Ecole Polytechnique Fédérale de Lausanne, 1015 Lausanne, Switzerland; email : Jean-Francois.Scheid@epfl.ch
} 
In the next section, we give a sketch of the modelling leading to problem $(P)$. This derivation is based on [12] and [7]. We establish the well-posedness of problem $(P)$ by first assuming that the nonlinear terms $F_{i}$ and $D_{i}, i=1,2$ are bounded and Lipschitz functions. Then, under these assumptions, we prove the existence of a weak solution in Section 3 by the use of a Faedo-Galerkin method. Next, regularity and uniqueness results are investigated in Section 4. Finally, we obtain in Section 5 a maximum principle under the additional assumptions that the nonlinear functions $F_{1}, F_{2}$ and $D_{2}$ vanish beyond the sets $\{0<c<1\}$ and $\{0<\phi<1\}$. This maximum principle ensures that $c$ and $\phi$ stay between 0 and 1 so long as the initial data $c_{0}$ and $\phi_{0}$ do. Then this justifies the assumptions made in Section 3 about the boundeness and Lipschitz conditions for the nonlinear terms.

\section{Modelling}

This section is devoted to a brief introduction of the derivation of the phase-field model we study. For a complete description of the modelling, we refer to [12], [7].

Let us consider an alloy of two components $A$ and $B$ in a spacial domain $\Omega$ of $\mathbb{R}^{d}$ with $d \leq 3$. The system is on the one-hand caracterized by the relative concentration $c=c(x, t)$ of the component $B$ with respect to the mixture, for $x \in \Omega$ and at time $t$. The function $c$ is then always between 0 and 1 . On the other-hand, the solidification state of the alloy is taken into account by an order parameter $\phi=\phi(x, t)$, the so-called phase-field. More precisely, the phase-field equals 0 if the system is in a solid phase and equals 1 if it is in a liquid phase, but may take continuous values between 0 and 1 .

We suppose that the solidification process takes place at a constant and homogeneous temperature $T$ which is fixed somewhere between the two melting temperatures of the components $A$ and $B$. To have a thermodynamical description of the system, we introduce a GinzburgLandau free energy functional by using a free energy density $f$ which depends on $c$ and $\phi$. The thermodynamical laws together with the mass conservation law imply the following equations for $\phi$ and $c$ (see [12], [7]) :

$$
\begin{aligned}
\alpha \frac{\partial \phi}{\partial t} & =\varepsilon^{2} \Delta \phi-\frac{\partial f}{\partial \phi} \quad \text { in } \Omega, \\
\frac{\partial c}{\partial t} & =\operatorname{div}\left(M \nabla \frac{\partial f}{\partial c}\right) \quad \text { in } \Omega
\end{aligned}
$$

where $\varepsilon$ and $\alpha$ are positive parameters and $M=M(c, \phi)$ is a positive function.

Moreover, $c$ and $\phi$ are subject to homogeneous Neumann boundary conditions on the boundary $\partial \Omega$ of $\Omega$.

Using some basic thermodynamical principles, we can infer (see [12], [7]) that

$$
-\frac{\partial f}{\partial \phi}=F_{1}(\phi)+c F_{2}(\phi)
$$

where $F_{1}, F_{2}$ are functions of $\phi$ that vanish for $\phi=0$ and $\phi=1$, and

$$
\nabla \frac{\partial f}{\partial c}=-F_{2}(\phi) \nabla \phi+\frac{R T}{v_{m}} \frac{1}{c(1-c)} \nabla c
$$

where $R$ is the Boltzman constant and $v_{m}$ the molar volume.

We point out that $F_{1}$ and $F_{2}$ can be choosen as polynomial functions of 4 th degree that guarantee some thermodynamical constraints. 
Hence, equation (1) with (3) becomes

$$
\alpha \frac{\partial \phi}{\partial t}=\varepsilon^{2} \Delta \phi+F_{1}(\phi)+c F_{2}(\phi) \text { in } \Omega .
$$

The parameters $\alpha$ and $\varepsilon^{2}$ can be related to physical parameters by exhibiting a special solution for the phase-field in a one-dimensional case for a pure element ( $c \equiv 0$ or $c \equiv 1)$ and performing an asymptotic analysis as $\varepsilon$ tends towards 0 (see [12], [7], [6]). In that way, $\varepsilon^{2}$ is proportional to the interface thickness.

Moreover, in order to recover a classical diffusion equation for $c$ whenever $\phi \equiv 0$ or $\phi \equiv 1$ i.e. in a totally solid or liquid phase, we choose

$$
M(c, \phi)=\frac{v_{m}}{R T} D_{1}(\phi) c(1-c),
$$

where $D_{1}$ is an increasing smooth function such that $D_{1}(0)>0$ and $D_{1}(1)>0$ are respectively the solid and liquid diffusion coefficients, and $D_{1}$ is bounded above and below by two positive constants $D_{s}<D_{l}$.

Thus, equation (2) with (4) and (6) leads to

$$
\frac{\partial c}{\partial t}=\operatorname{div}\left(D_{1}(\phi) \nabla c+D_{2}(c, \phi) \nabla \phi\right) \quad \text { in } \Omega,
$$

where $D_{2}(c, \phi)=-\frac{v_{m}}{R T} D_{1}(\phi) c(1-c) F_{2}(\phi)$ is a smooth function vanishing for $c=0$ and $c=1$.

Without loss of generality for the purpose of this article, we may choose $\alpha=1$ and then the equations (5) and (7) together with homogeneous Neumann boundary conditions and initial conditions lead to problem $(P)$.

Notations and assumptions. Throughout the article, we assume that $\Omega$ is an open bounded domain in $\mathbb{R}^{d}, d \leq 3$ with a smooth boundary $\partial \Omega$ of class $C^{\infty}$. We note $V=H^{1}(\Omega)$ and $V^{\prime}$ is the dual space $\left(H^{1}(\Omega)\right)^{\prime}$ of $H^{1}(\Omega)$. We denote by $<., .>_{V^{\prime}, V}$ the duality product between $V^{\prime}$ and $V$ and we also denote $Q_{T}=\Omega \times(0, T)$, for $T>0$.

\section{Existence results.}

In this section, we prove the existence of a weak solution to problem $(P)$ under Lipschitz and boundeness assumptions on the nonlinear functions $F_{i}$ and $D_{i}, i=1,2$. More precisely, we assume that

(H1) $\cdot F_{1}, F_{2} \in C(\mathbb{R})$ are Lipschitz and bounded functions with

$$
\left|F_{i}(r)\right| \leq M_{1} \quad \text { for } i=1,2 \text { and } \forall r \in \mathbb{R} .
$$

(H2) - $D_{1} \in C(\mathbb{R})$ is a Lipschitz positive and bounded function with

$$
0<D_{s} \leq D_{1}(r) \leq D_{l}, \quad \forall r \in \mathbb{R} .
$$

(H3) - $D_{2} \in C(\mathbb{R} \times \mathbb{R})$ is a Lipschitz and bounded function with

$$
\left|D_{2}\left(r_{1}, r_{2}\right)\right| \leq M_{2}, \quad \forall\left(r_{1}, r_{2}\right) \in \mathbb{R} \times \mathbb{R} .
$$

Before expressing and proving the existence result relative to the previous assumptions, we recall a well-known result from elliptic theory (see for instance [9], Chap. 2, Th. 5.1) that will be useful later on. 
Lemma 1 Let $k \in \mathbb{N}$ and $u \in H^{2}(\Omega)$ satisfying $\Delta u \in H^{k}(\Omega)$ and $\frac{\partial u}{\partial n}=0$ on $\partial \Omega$.

Then $u \in H^{k+2}(\Omega)$ and there exists a constant $C>0$ independent of $u$ such that

$$
\|u\|_{H^{k+2}(\Omega)} \leq C\left(\|\Delta u\|_{H^{k}(\Omega)}+\|u\|_{H^{k}(\Omega)}\right) .
$$

Now, we state the main result of this section :

Theorem 1 Let assumptions (H1)-(H3) be fulfilled.

1) For any $\left(\phi_{0}, c_{0}\right) \in L^{2}(\Omega) \times L^{2}(\Omega)$ and $T>0$, there exists a couple of functions $(\phi, c)$ satisfying

$$
\phi, c \in L^{2}\left(0, T ; H^{1}(\Omega)\right) \cap H^{1}\left(0, T ; V^{\prime}\right),
$$

such that $\phi(0)=\phi_{0}, c(0)=c_{0}$ and

$$
\begin{aligned}
& <\frac{\partial \phi}{\partial t}, v>_{V^{\prime}, V}+\varepsilon^{2} \int_{\Omega} \nabla \phi \cdot \nabla v d x=\int_{\Omega}\left(F_{1}(\phi)+c F_{2}(\phi)\right) v d x, \\
& <\frac{\partial c}{\partial t}, w>_{V^{\prime}, V}+\int_{\Omega}\left(D_{1}(\phi) \nabla c+D_{2}(c, \phi) \nabla \phi\right) \cdot \nabla w d x=0
\end{aligned}
$$

for all $v, w \in H^{1}(\Omega)$ and a.e. in $(0, T)$.

2) For any $\left(\phi_{0}, c_{0}\right) \in H^{1}(\Omega) \times L^{2}(\Omega)$ and $T>0$, there exists a couple of functions $(\phi, c)$ satisfying

$$
\begin{aligned}
& \phi \in L^{2}\left(0, T ; H^{2}(\Omega)\right) \cap H^{1}\left(0, T ; L^{2}(\Omega)\right), \\
& c \in L^{2}\left(0, T ; H^{1}(\Omega)\right) \cap H^{1}\left(0, T ; V^{\prime}\right),
\end{aligned}
$$

such that $\phi(0)=\phi_{0}, c(0)=c_{0}$ and

$$
\begin{aligned}
& \frac{\partial \phi}{\partial t}-\varepsilon^{2} \Delta \phi=F_{1}(\phi)+c F_{2}(\phi) \quad \text { a.e. in } Q_{T}, \\
& \frac{\partial \phi}{\partial n}=0 \quad \text { a.e. on } \partial \Omega \times(0, T), \\
& <\frac{\partial c}{\partial t}, v>_{V^{\prime}, V}+\int_{\Omega}\left(D_{1}(\phi) \nabla c+D_{2}(c, \phi) \nabla \phi\right) \cdot \nabla v d x=0, \\
& \text { for all } v \in H^{1}(\Omega) \text { and a.e. in }(0, T) \text {. }
\end{aligned}
$$

REMARK : Since $\phi, c \in L^{2}\left(0, T ; H^{1}(\Omega)\right) \cap H^{1}\left(0, T ; V^{\prime}\right)$ it follows that $\phi, c \in C\left([0, T] ; L^{2}(\Omega)\right)$. Moreover, as soon as $\phi \in L^{2}\left(0, T ; H^{2}(\Omega)\right) \cap H^{1}\left(0, T ; L^{2}(\Omega)\right)$, we infer that $\phi \in C\left([0, T] ; H^{1}(\Omega)\right)$ (see for instance [9], Th. 3.1, p. 23).

Proof of part 1) of Theorem 1 : We employ a Faedo-Galerkin method. We consider the se-

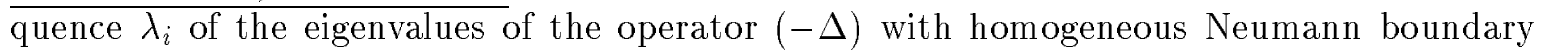
conditions such that

$$
0=\lambda_{1}<\lambda_{2} \leq \ldots \leq \lambda_{k} \leq \ldots,
$$

and the corresponding eigenfunctions $\left\{v_{j}\right\}_{j \geq 1}$ such that $\left(v_{j}, v_{k}\right)_{L^{2}(\Omega)}=\delta_{j k}$ for $1 \leq j, k$. Note that the eigenfunctions satisfy $\left(\nabla v_{j}, \nabla v_{k}\right)_{L^{2}(\Omega)}=0$ for $j \neq k, 1 \leq j, k$. Moreover, the eigenfunctions are smooth functions and $\left\{v_{j}\right\}_{j \geq 1}$ form a complete orthogonal basis in $L^{2}(\Omega)$ and in $H^{1}(\Omega)$. We denote by $V_{m}$ the finite vector space spanned by $\left\{v_{j}\right\}_{1 \leq j \leq m}$. Then $\underset{m \geq 1}{\cup} V_{m}$ 
is dense in $L^{2}(\Omega)$ and also in $H^{1}(\Omega)$. In the sequel, we will make use of the $L^{2}$-orthogonal projector $p_{m}$ on the space $V_{m}$. Note that we have, for all $\varphi \in H^{1}(\Omega)$

$$
\left(\nabla\left(p_{m} \varphi-\varphi\right), \nabla v\right)_{L^{2}(\Omega)}=0 \quad \forall v \in V_{m},
$$

and then $p_{m}$ is also the $H^{1}$-orthogonal projector on $V_{m}$.

For each $m \geq 1$, we then consider the approximate problem of finding $\phi_{m}, c_{m} \in H^{1}\left(0, T ; V_{m}\right)$ satisfying

$$
\left(P_{m}\right)\left\{\begin{array}{l}
\int_{\Omega} \frac{\partial \phi_{m}}{\partial t} v d x+\varepsilon^{2} \int_{\Omega} \nabla \phi_{m} \cdot \nabla v d x=\int_{\Omega}\left(F_{1}\left(\phi_{m}\right)+c_{m} F_{2}\left(\phi_{m}\right)\right) v d x \\
\int_{\Omega} \frac{\partial c_{m}}{\partial t} w d x+\int_{\Omega}\left(D_{1}\left(\phi_{m}\right) \nabla c_{m}+D_{2}\left(c_{m}, \phi_{m}\right) \nabla \phi_{m}\right) \cdot \nabla w d x=0 \\
\quad \text { for all } v, w \in V_{m} \text { and for a.e. } t \in(0, T) \\
\phi_{m}(0)=\phi_{0 m} \in V_{m}, \quad c_{m}(0)=c_{0 m} \in V_{m} .
\end{array}\right.
$$

We choose $\phi_{0 m}=p_{m} \phi_{0}$ and $c_{0 m}=p_{m} c_{0}$. Then, the initial data have the following properties

$$
\begin{gathered}
\left\|\phi_{0 m}\right\|_{L^{2}(\Omega)} \leq\left\|\phi_{0}\right\|_{L^{2}(\Omega)} \quad \text { and } \quad \phi_{0 m} \rightarrow \phi_{0} \text { in } L^{2}(\Omega) \text { as } m \rightarrow+\infty, \\
\left\|c_{0 m}\right\|_{L^{2}(\Omega)} \leq\left\|c_{0}\right\|_{L^{2}(\Omega)} \quad \text { and } \quad c_{0 m} \rightarrow c_{0} \text { in } L^{2}(\Omega) \text { as } m \rightarrow+\infty .
\end{gathered}
$$

We look for an approximate solution $\left(\phi_{m}, c_{m}\right)$ of the form

$$
\phi_{m}(t)=\sum_{i=1}^{m} \varphi_{i m}(t) v_{i}, \quad c_{m}(t)=\sum_{i=1}^{m} c_{i m}(t) v_{i},
$$

so that Problem $\left(P_{m}\right)$ is in fact an initial value problem for a system of $2 m$ ordinary differential equations which can read as

$$
\left\{\begin{array}{l}
U_{m}^{\prime}(t)+B\left(U_{m}(t)\right) U_{m}(t)=\mathcal{F}\left(U_{m}(t)\right) \\
U_{m}(0)=U_{0}
\end{array}\right.
$$

where $U_{m}(t)=\left(\begin{array}{c}\left(\varphi_{i m}(t)\right) \\ \left(c_{i m}(t)\right)\end{array}\right) \in \mathbb{R}^{2 m}$.

Since the nonlinear terms $F_{1}, F_{2}, D_{1}$ and $D_{2}$ are Lipschitz continuous functions and since $\left(v_{j}\right)$ are smooth functions, the above problem has a unique solution $U_{m}(t)$ on some maximal time interval $\left[0, T_{m}\right)$ with $T_{m}>0$ and $U_{m} \in C^{1}\left(\left[0, T_{m}\right)\right)$. Hence Problem $\left(P_{m}\right)$ has a unique solution $\left(\phi_{m}, c_{m}\right)$ on the time interval $\left[0, T_{m}\right)$ and $\left(\phi_{m}, c_{m}\right) \in C^{1}\left(\left[0, T_{m}\right), V_{m} \times V_{m}\right)$.

Next we derive some estimates which show in particular that $T_{m}=+\infty$. From now on, we will denote by $C$ a positive constant which depends on $\varepsilon^{2}, M_{1}, M_{2}, D_{s}, D_{l},|\Omega|, T$, on the Lipschitz constants of nonlinear terms $F_{i}, D_{i}, i=1,2$ and also on $\left\|\phi_{0}\right\|_{L^{2}(\Omega)}$ and $\left\|c_{0}\right\|_{L^{2}(\Omega)}$ but which is independent of $m$.

\section{A priori estimates.}

i) We choose $v=\phi_{m}$ in (12) and $w=c_{m}$ in (13) to obtain

$$
\begin{aligned}
& \frac{1}{2} \frac{d}{d t} \int_{\Omega} \phi_{m}^{2}+\varepsilon^{2} \int_{\Omega}\left|\nabla \phi_{m}\right|^{2}=\int_{\Omega} F_{1}\left(\phi_{m}\right) \phi_{m}+\int_{\Omega} F_{2}\left(\phi_{m}\right) c_{m} \phi_{m} \\
& \frac{1}{2} \frac{d}{d t} \int_{\Omega} c_{m}^{2}+\int_{\Omega} D_{1}\left(\phi_{m}\right)\left|\nabla c_{m}\right|^{2}=-\int_{\Omega} D_{2}\left(c_{m}, \phi_{m}\right) \nabla \phi_{m} \cdot \nabla c_{m}
\end{aligned}
$$


Multiplying (18) by $\delta>0$ that will be choosen later, adding it to (17) and using also (H1) - (H3), we have

$$
\begin{aligned}
\frac{1}{2} \frac{d}{d t} \int_{\Omega}\left(\phi_{m}^{2}\right. & \left.+\delta c_{m}^{2}\right)+\varepsilon^{2} \int_{\Omega}\left|\nabla \phi_{m}\right|^{2}+\delta D_{s} \int_{\Omega}\left|\nabla c_{m}\right|^{2} \\
& \leq M_{1}\left(\int_{\Omega}\left|\phi_{m}\right|+\int_{\Omega}\left|c_{m}\right|\left|\phi_{m}\right|\right)+M_{2} \delta \int_{\Omega}\left|\nabla \phi_{m}\right|\left|\nabla c_{m}\right| \\
& \leq C\left(1+\int_{\Omega} \phi_{m}^{2}+\int_{\Omega} c_{m}^{2}\right)+\frac{M_{2} \delta}{2 \eta} \int_{\Omega}\left|\nabla \phi_{m}\right|^{2}+\frac{M_{2} \delta}{2} \eta \int_{\Omega}\left|\nabla c_{m}\right|^{2},
\end{aligned}
$$

for all $\eta>0$, by the use of Young's inequality. Choosing $\delta=D_{s} \frac{\varepsilon^{2}}{M_{2}^{2}}$ and $\eta=\frac{D_{s}}{M_{2}}$, we get

$$
\frac{d}{d t} \int_{\Omega}\left(\phi_{m}^{2}+\delta c_{m}^{2}\right)+\varepsilon^{2} \int_{\Omega}\left|\nabla \phi_{m}\right|^{2}+\delta D_{s} \int_{\Omega}\left|\nabla c_{m}\right|^{2} \leq 2 C\left(1+\int_{\Omega} \phi_{m}^{2}+\int_{\Omega} c_{m}^{2}\right) .
$$

It follows by Gronwall's lemma and (14), (15), (16) that there exists a constant $C>0$ depending on $T_{m}$ such that, for all $0 \leq t<T_{m}$

$$
\left\|\phi_{m}(t)\right\|_{L^{2}(\Omega)}+\left\|c_{m}(t)\right\|_{L^{2}(\Omega)} \leq C .
$$

The estimate (20) shows that $T_{m}=+\infty$. Moreover, we can see that if $t$ belongs to the fixed interval $[0, T]$, then the estimate $(20)$ holds with a constant $C$ independent of $m$. Integrating inequality (19) from 0 to $T$ and using (20), (19), we conclude there exists a constant $C$ such that

$$
\begin{aligned}
\left\|\left(c_{m}, \phi_{m}\right)\right\|_{\left(L^{2}\left(0, T ; H^{1}(\Omega)\right)\right)^{2}} & \leq C, \\
\left\|\left(c_{m}, \phi_{m}\right)\right\|_{\left(L^{\infty}\left(0, T ; L^{2}(\Omega)\right)\right)^{2}} & \leq C .
\end{aligned}
$$

ii) Let us now give estimates of the time derivatives of $\phi_{m}$ and $c_{m}$. Due to the fact that the $L^{2}$-orthogonal projector $p_{m}$ is also the $H^{1}(\Omega)$-orthogonal projector on $V_{m}$, we have for $\varphi_{m} \in V_{m}$

$$
\left\|\varphi_{m}\right\|_{V^{\prime}} \equiv \sup _{\substack{\psi \in V \\ \psi \neq 0}} \frac{<\varphi_{m}, \psi>_{V^{\prime}, V}}{\|\psi\|_{V}}=\max _{\substack{\psi_{m} \in V_{m} \\ \psi_{m} \neq 0}} \frac{\left(\varphi_{m}, \psi_{m}\right)_{L^{2}(\Omega)}}{\left\|\psi_{m}\right\|_{V}} .
$$

Thus, from equation (12), we infer that for all $0 \leq t \leq T$

$$
\left\|\frac{\partial \phi_{m}(t)}{\partial t}\right\|_{V^{\prime}} \leq \varepsilon^{2}\left\|\nabla \phi_{m}(t)\right\|_{L^{2}(\Omega)}+C\left(1+\left\|c_{m}(t)\right\|_{L^{2}(\Omega)}\right)
$$

and then

$$
\left\|\frac{\partial \phi_{m}}{\partial t}\right\|_{L^{2}\left(0, T ; V^{\prime}\right)} \leq C\left(1+\left\|c_{m}\right\|_{L^{2}\left(Q_{T}\right)}+\left\|\phi_{m}\right\|_{L^{2}\left(0, T ; H^{1}(\Omega)\right)}\right) .
$$

In an analogous way, we obtain the following estimate for the time derivative of $c_{m}$ :

$$
\left\|\frac{\partial c_{m}}{\partial t}\right\|_{L^{2}\left(0, T ; V^{\prime}\right)} \leq C\left(\left\|c_{m}\right\|_{L^{2}\left(0, T ; H^{1}(\Omega)\right)}+\left\|\phi_{m}\right\|_{L^{2}\left(0, T ; H^{1}(\Omega)\right)}\right) .
$$

Thus, using estimate (21) in (23) and (24), we get that

$$
\left\|\left(\frac{\partial c_{m}}{\partial t}, \frac{\partial \phi_{m}}{\partial t}\right)\right\|_{\left(L^{2}\left(0, T ; V^{\prime}\right)\right)^{2}} \leq C .
$$


Taking the limit.

We deduce from (21), (22) and (25) that $c_{m}$ and $\phi_{m}$ are bounded (uniformly with respect to $m$ ) in

$$
W_{1}=\left\{u \in L^{2}\left(0, T ; H^{1}(\Omega)\right), \quad \frac{\partial u}{\partial t} \in L^{2}\left(0, T ; V^{\prime}\right)\right\}
$$

and in

$$
W_{2}=\left\{u \in L^{\infty}\left(0, T ; L^{2}(\Omega)\right), \quad \frac{\partial u}{\partial t} \in L^{2}\left(0, T ; V^{\prime}\right)\right\} .
$$

Since the embedding of $H^{1}(\Omega)$ into $L^{2}(\Omega)$ is compact, $W_{1}$ is compactly embedded into $L^{2}\left(Q_{T}\right)$ (see for instance [11], Chap. 3, Th. 2.1). Moreover, due to the compacteness of the embedding of $L^{2}(\Omega)$ into $V^{\prime}$, the space $W_{2}$ is compactly embedded into $C\left([0, T], V^{\prime}\right)$ (see [10], Cor. 4). Hence, there exist

$$
c, \phi \in L^{2}\left(0, T ; H^{1}(\Omega)\right) \cap H^{1}\left(0, T ; V^{\prime}\right)
$$

and subsequences of $c_{m}$ and $\phi_{m}$ (we still denote $c_{m}$ and $\phi_{m}$ ) such that, as $m \rightarrow+\infty$,

$$
\begin{array}{ll}
\left(c_{m}, \phi_{m}\right) \rightarrow(c, \phi) & \text { in }\left(L^{2}\left(0, T ; H^{1}(\Omega)\right)\right)^{2} \text { weakly, } \\
\left(c_{m}, \phi_{m}\right) \rightarrow(c, \phi) & \text { in }\left(L^{2}\left(Q_{T}\right)\right)^{2} \cap\left(C\left([0, T], V^{\prime}\right)\right)^{2} \text { strongly, } \\
\left(\frac{\partial c_{m}}{\partial t}, \frac{\partial \phi_{m}}{\partial t}\right) \rightarrow\left(\frac{\partial c}{\partial t}, \frac{\partial \phi}{\partial t}\right) & \text { in }\left(L^{2}\left(0, T, V^{\prime}\right)\right)^{2} \text { weakly. }
\end{array}
$$

In order to take the limit in Problem $\left(P_{m}\right)$, we need the following convergence results for the nonlinear terms.

\section{Lemma 2}

$$
\begin{array}{ll}
F_{i}\left(\phi_{m}\right) \rightarrow F_{i}(\phi) \quad i=1,2 & \text { in } L^{p}\left(Q_{T}\right), \forall p \in[1,+\infty), \\
D_{1}\left(\phi_{m}\right) \rightarrow D_{1}(\phi) & \text { in } L^{p}\left(Q_{T}\right), \forall p \in[1,+\infty), \\
c_{m} F_{2}\left(\phi_{m}\right) \rightarrow c F_{2}(\phi) & \text { in } L^{q}\left(Q_{T}\right), \forall q \in[1,2), \\
D_{1}\left(\phi_{m}\right) \nabla c_{m} \rightarrow D_{1}(\phi) \nabla c & \text { in } L^{q}\left(Q_{T}\right) \text { weakly, } \forall q \in[1,2), \\
D_{2}\left(c_{m}, \phi_{m}\right) \nabla \phi_{m} \rightarrow D_{2}(c, \phi) \nabla \phi & \text { in } L^{q}\left(Q_{T}\right) \text { weakly, } \forall q \in[1,2) .
\end{array}
$$

Proof of Lemma 2: The convergences in (29), (30) and (31) are obtained using the fact that $\phi_{m} \rightarrow \phi$ and $c_{m} \rightarrow c$ in $L^{2}\left(Q_{T}\right)$ strongly and the assumptions (H1), (H2) on $F_{i}$ and $D_{1}$. The convergences in (32) and (33) required the weak convergences (26) but the strong convergence (27) is necessary to obtain the convergence in (33). We only give details for the proof of (33).

Let $v \in L^{q^{*}}\left(Q_{T}\right)$ with $\frac{1}{q^{*}}+\frac{1}{q}=1, q \in[1,2)$ and consider

$$
\mathcal{A}_{m}=\int_{Q_{T}}\left(D_{2}\left(c_{m}, \phi_{m}\right) \nabla \phi_{m}-D_{2}(c, \phi) \nabla \phi\right) v d x d t
$$

Then we write $\mathcal{A}_{m}$ in the following form

$$
\mathcal{A}_{m}=\int_{Q_{T}} D_{2}(c, \phi)\left(\nabla \phi_{m}-\nabla \phi\right) v d x d t+\int_{Q_{T}}\left(D_{2}\left(c_{m}, \phi_{m}\right)-D_{2}(c, \phi)\right) \nabla \phi_{m} v d x d t
$$


The first term tends to 0 as $m \rightarrow 0$ thanks to $(26)$ and to the fact that $D_{2}(c, \phi) v \in L^{2}\left(Q_{T}\right)$. Let $\mathcal{A}^{\prime}{ }_{m}$ denote the second term in the expression of $\mathcal{A}_{m}$. Then, using Hölder's inequality, we have

$$
\left|\mathcal{A}_{m}^{\prime}\right| \leq\left\|D_{2}\left(c_{m}, \phi_{m}\right)-D_{2}(c, \phi)\right\|_{L^{s}\left(Q_{T}\right)}\left\|\nabla \phi_{m}\right\|_{L^{2}\left(Q_{T}\right)}\|v\|_{L^{q^{*}}\left(Q_{T}\right)},
$$

with $\frac{1}{q^{*}}+\frac{1}{2}+\frac{1}{s}=1$. Moreover, since $s \geq 2$ and $D_{2}$ is a bounded Lipschitz function, it follows that

$$
\left\|D_{2}\left(c_{m}, \phi_{m}\right)-D_{2}(c, \phi)\right\|_{L^{s}\left(Q_{T}\right)}^{s} \leq C\left(\left\|c_{m}-c\right\|_{L^{2}\left(Q_{T}\right)}^{2}+\left\|\phi_{m}-\phi\right\|_{L^{2}\left(Q_{T}\right)}^{2}\right) .
$$

We then show that $\mathcal{A}_{m} \rightarrow 0$ by the use of (27) and (25).

We now take the limit in Problem $\left(P_{m}\right)$ and find that $c$ and $\phi$ satisfy equations (9) for all $v, w \in H^{1}(\Omega)$. Moreover, we conclude from (14), (15), (16) and (27) that $\phi(0)=\phi_{0}$ and $c(0)=c_{0}$.

Proof of part 2) of Theorem 1: We suppose now that $\phi_{0} \in H^{1}(\Omega)$. Since we chose $\phi_{0 m}=p_{m} \phi_{0}$ $\overline{\text { as initial data for Problem }\left(P_{m}\right)}$, we deduce from the property (11) about $p_{m}$, that

$$
\left\|\nabla \phi_{0 m}\right\|_{L^{2}(\Omega)} \leq\left\|\nabla \phi_{0}\right\|_{L^{2}(\Omega)} .
$$

We derive then some new estimates for $\phi_{m}$. From now on, we still denote by $C$ a positive constant as in the proof of part 1), but it depends now also on $\left\|\nabla \phi_{0}\right\|_{L^{2}(\Omega)}$.

i) First, let us choose $v=\frac{\partial \phi_{m}}{\partial t}$ in (12). We obtain

$$
\int_{\Omega}\left|\frac{\partial \phi_{m}}{\partial t}\right|^{2}+\varepsilon^{2} \int_{\Omega} \nabla \phi_{m} \cdot \nabla \frac{\partial \phi_{m}}{\partial t}=\int_{\Omega} F_{1}\left(\phi_{m}\right) \frac{\partial \phi_{m}}{\partial t}+\int_{\Omega} F_{2}\left(\phi_{m}\right) c_{m} \frac{\partial \phi_{m}}{\partial t} .
$$

Using (H1) and Young's inequality, we have for all $\eta_{1}, \eta_{2}>0$ :

$$
\int_{\Omega}\left|\frac{\partial \phi_{m}}{\partial t}\right|^{2}+\frac{\varepsilon^{2}}{2} \frac{d}{d t} \int_{\Omega}\left|\nabla \phi_{m}\right|^{2} \leq \eta_{1} M_{1}|\Omega|+\left(\frac{M_{1}}{4 \eta_{1}}+\frac{M_{2}}{4 \eta_{2}}\right) \int_{\Omega}\left|\frac{\partial \phi_{m}}{\partial t}\right|^{2}+M_{2} \eta_{2} \int_{\Omega}\left|c_{m}\right|^{2} .
$$

We choose $\eta_{1}=M_{1}$ and $\eta_{2}=M_{2}$ so that we obtain

$$
\int_{\Omega}\left|\frac{\partial \phi_{m}}{\partial t}\right|^{2}+\varepsilon^{2} \frac{d}{d t} \int_{\Omega}\left|\nabla \phi_{m}\right|^{2} \leq C\left(1+\int_{\Omega}\left|c_{m}\right|^{2}\right) .
$$

Integrating this inequality over $(0, t)$ for $t \in(0, T)$, we get

$$
\int_{0}^{t} \int_{\Omega}\left|\frac{\partial \phi_{m}}{\partial t}\right|^{2}+\varepsilon^{2} \int_{\Omega}\left|\nabla \phi_{m}(t)\right|^{2} \leq C\left(t+\int_{0}^{t} \int_{\Omega}\left|c_{m}\right|^{2}\right)+\varepsilon^{2} \int_{\Omega}\left|\nabla \phi_{0}\right|^{2} .
$$

Hence, thanks to (21) we conclude there exists $C$ such that

$$
\left\|\frac{\partial \phi_{m}}{\partial t}\right\|_{L^{2}\left(Q_{T}\right)}+\left\|\phi_{m}\right\|_{L^{\infty}\left(0, T ; H^{1}(\Omega)\right)} \leq C
$$

ii) Finally, by remarking that $-\Delta \phi_{m} \in V_{m}$, we choose $v=-\Delta \phi_{m}$ in (12). Integrating by parts the two terms in the resulting left hand-side and using the fact that $\phi_{m}$ satisfies the homogeneous Neumann boundary condition on $\partial \Omega$, we obtain

$$
\frac{1}{2} \frac{d}{d t} \int_{\Omega}\left|\nabla \phi_{m}\right|^{2}+\varepsilon^{2} \int_{\Omega}\left|\Delta \phi_{m}\right|^{2}=-\int_{\Omega} F_{1}\left(\phi_{m}\right) \Delta \phi_{m}-\int_{\Omega} F_{2}\left(\phi_{m}\right) c_{m} \Delta \phi_{m}
$$


Using (H1) and Young's inequality, we get for all $\eta_{1}, \eta_{2}>0$

$$
\frac{1}{2} \frac{d}{d t} \int_{\Omega}\left|\nabla \phi_{m}\right|^{2}+\varepsilon^{2} \int_{\Omega}\left|\Delta \phi_{m}\right|^{2} \leq \eta_{1} M_{1}|\Omega|+\left(\frac{M_{1}}{4 \eta_{1}}+\frac{M_{2}}{4 \eta_{2}}\right) \int_{\Omega}\left|\Delta \phi_{m}\right|^{2}+M_{2} \eta_{2} \int_{\Omega}\left|c_{m}\right|^{2} .
$$

Then choosing $\eta_{1}=\frac{M_{1}}{\varepsilon^{2}}$ and $\eta_{2}=\frac{M_{2}}{\varepsilon^{2}}$, it follows there exists $C$ such that

$$
\frac{d}{d t} \int_{\Omega}\left|\nabla \phi_{m}\right|^{2}+\varepsilon^{2} \int_{\Omega}\left|\Delta \phi_{m}\right|^{2} \leq C\left(1+\int_{\Omega}\left|c_{m}\right|^{2}\right)
$$

Integrating this inequality from 0 to $T$ and using (21), we obtain that

$$
\left\|\Delta \phi_{m}\right\|_{L^{2}\left(Q_{T}\right)} \leq C
$$

From the elliptic estimate (8) of Lemma 1, combined with estimates (21) and (36), we conclude that

$$
\left\|\phi_{m}\right\|_{L^{2}\left(0, T ; H^{2}(\Omega)\right)} \leq C
$$

We deduce from (35) and (37) that when $\phi_{0} \in H^{1}(\Omega), \phi_{m}$ is bounded (uniformely with respect to $m$ ) in

$$
W_{3}=\left\{u \in L^{2}\left(0, T ; H^{2}(\Omega)\right), \quad \frac{\partial u}{\partial t} \in L^{2}\left(0, T ; L^{2}(\Omega)\right)\right\} .
$$

Since the embedding of $H^{2}(\Omega)$ into $H^{1}(\Omega)$ is compact, we conclude (see for instance [11], Chap. 3, Th. 2.1) that $W_{3}$ is compactly embedded into $L^{2}\left(0, T ; H^{1}(\Omega)\right)$. By taking the limit $\phi$ of a subsequence of $\phi_{m}$, we can conclude the proof of part 2) of Theorem 1 in the same way for part 1). More precisely, we have

$$
\phi \in L^{2}\left(0, T ; H^{2}(\Omega)\right) \cap H^{1}\left(0, T ; L^{2}(\Omega)\right)
$$

and there exists a subsequence of $\phi_{m}$ (we still denote $\phi_{m}$ ) such that, as $m \rightarrow+\infty$,

$$
\begin{array}{ll}
\phi_{m} \rightarrow \phi & \text { in } L^{2}\left(0, T ; H^{1}(\Omega)\right) \text { strongly } \\
\frac{\partial \phi_{m}}{\partial t} \rightarrow \frac{\partial \phi}{\partial t} & \text { in } L^{2}\left(Q_{T}\right) \text { weakly. }
\end{array}
$$

We then pass to the limit in Problem $\left(P_{m}\right)$ and find that $c$ and $\phi$ satisfy $(10)$.

REMARK : Theorem 1 is valid for any smooth domain $\Omega \subset \mathbb{R}^{d}$ with $d \geq 1$. The restriction $d \leq 3$ does not occur in the proof.

\section{Regularity and uniqueness}

In this section, we prove a regularity and uniqueness result under the additional assumption that the initial data are smooth enough. To begin with, we recall some properties about Sobolev spaces that will be useful later on. 
Lemma 3 Let $\Omega \subset \mathbb{R}^{3}$ be an open and bounded set with a smooth boundary.

Then the following properties hold:

1) $H^{1}(\Omega) \hookrightarrow L^{q}(\Omega)$ for $1 \leq q \leq 6$ with continuous embedding.

2) Gagliardo-Nirenberg inequalities (see [5]).

There exist two constants $C_{1}>0$ and $C_{2}>0$ such that

$$
\begin{aligned}
& \|u\|_{L^{3}(\Omega)} \leq C_{1}\|u\|_{H^{1}(\Omega)}^{1 / 2}\|u\|_{L^{2}(\Omega)}^{1 / 2} \quad \text { for all } u \in H^{1}(\Omega) . \\
& \|u\|_{L^{6}(\Omega)} \leq C_{2}\|u\|_{H^{2}(\Omega)}^{1 / 2}\|u\|_{L^{2}(\Omega)}^{1 / 2} \quad \text { for all } u \in H^{2}(\Omega) .
\end{aligned}
$$

We state the following regularity and uniqueness result.

Theorem 2 Let assumptions (H1)-(H3) be fulfilled.

Let $\phi_{0} \in H^{2}(\Omega)$ such that $\frac{\partial \phi_{0}}{\partial n}=0$ on $\partial \Omega$ and $c_{0} \in H^{1}(\Omega)$. Then for any $T>0$, there exists a unique couple of functions $(\phi, c)$ satisfying

$$
\begin{aligned}
& \phi \in L^{2}\left(0, T ; H^{3}(\Omega)\right) \cap H^{1}\left(0, T ; H^{1}(\Omega)\right), \\
& c \in L^{2}\left(0, T ; H^{2}(\Omega)\right) \cap H^{1}\left(0, T ; L^{2}(\Omega)\right),
\end{aligned}
$$

such that $\phi(0)=\phi_{0}, c(0)=c_{0}$ and

$$
\begin{aligned}
& \frac{\partial \phi}{\partial t}-\varepsilon^{2} \Delta \phi=F_{1}(\phi)+c F_{2}(\phi) \quad \text { a.e. in } Q_{T}, \\
& \frac{\partial c}{\partial t}=\operatorname{div}\left(D_{1}(\phi) \nabla c+D_{2}(c, \phi) \nabla \phi\right) \quad \text { a.e. in } Q_{T}, \\
& \frac{\partial \phi}{\partial n}=\frac{\partial c}{\partial n}=0 \quad \text { a.e. on } \partial \Omega \times(0, T) .
\end{aligned}
$$

REMARK : We infer from the regularity of the solution (see e.g. [9]) that $\phi \in C\left([0, T] ; H^{2}(\Omega)\right.$ ) and $c \in C\left([0, T] ; H^{1}(\Omega)\right)$

Proof : a) Existence with regularity. To establish the regularity of a solution, we derive further a priori estimates in the Faedo-Galerkin procedure. Subsequently we will denote by $C$ a positive constant depending in particular on $\left\|\phi_{0}\right\|_{H^{2}(\Omega)}$ and $\left\|c_{0}\right\|_{H^{1}(\Omega)}$ but which is independent of $m$.

Additional a priori estimates for $\phi_{m}$.

i) First, we choose $v=\Delta^{2} \phi_{m} \in V_{m}$ in equation (12) of the approximate problem $\left(P_{m}\right)$. From the assumption (H1) we have that $F_{1}, F_{2} \in W^{1, \infty}(\mathbb{R})$. Then, integrating by parts the equation resulting from the specific choice of the test function and owing to the homogeneous Neumann boundary condition satisfied by $\frac{\partial \phi_{m}}{\partial t}$ and $\Delta \phi_{m}$, we obtain

$$
\frac{1}{2} \frac{d}{d t}\left\|\Delta \phi_{m}\right\|_{L^{2}(\Omega)}^{2}+\varepsilon^{2}\left\|\nabla \Delta \phi_{m}\right\|_{L^{2}(\Omega)}^{2}=\mathcal{F}_{m}
$$

where $\mathcal{F}_{m}=-\int_{\Omega}\left(F_{1}^{\prime}\left(\phi_{m}\right) \nabla \phi_{m}+F_{2}\left(\phi_{m}\right) \nabla c_{m}+F_{2}^{\prime}\left(\phi_{m}\right) c_{m} \nabla \phi_{m}\right) \cdot \nabla \Delta \phi_{m} d x$. 
We estimate $\mathcal{F}_{m}$ as follows. Using Young's inequality, we get the existence of a constant $C$ such that

$$
\mathcal{F}_{m} \leq \frac{\varepsilon^{2}}{4}\left\|\nabla \Delta \phi_{m}\right\|_{L^{2}(\Omega)}^{2}+C\left(\left\|\nabla \phi_{m}\right\|_{L^{2}(\Omega)}^{2}+\left\|\nabla c_{m}\right\|_{L^{2}(\Omega)}^{2}+\int_{\Omega}\left|c_{m} \nabla \phi_{m} \cdot \nabla \Delta \phi_{m}\right| d x\right) .
$$

The term $\mathcal{B}_{m}=\int_{\Omega}\left|c_{m} \nabla \phi_{m} . \nabla \Delta \phi_{m}\right| d x$ appearing in (47) is estimated by Hölder's inequality and the Gagliardo-Nirenberg inequalities (42)-(43) :

$$
\begin{aligned}
\mathcal{B}_{m} & \leq\left\|c_{m}\right\|_{L^{3}(\Omega)}\left\|\nabla \phi_{m}\right\|_{L^{6}(\Omega)}\left\|\nabla \Delta \phi_{m}\right\|_{L^{2}(\Omega)} \\
& \leq C_{1} C_{2}\left\|c_{m}\right\|_{H^{1}(\Omega)}^{1 / 2}\left\|c_{m}\right\|_{L^{2}(\Omega)}^{1 / 2}\left\|\nabla \phi_{m}\right\|_{H^{2}(\Omega)}^{1 / 2}\left\|\nabla \phi_{m}\right\|_{L^{2}(\Omega)}^{1 / 2}\left\|\nabla \Delta \phi_{m}\right\|_{L^{2}(\Omega)}
\end{aligned}
$$

Using (22) and (35), i.e. the fact that $c_{m}$ is bounded in $L^{\infty}\left(0, T ; L^{2}(\Omega)\right)$ and $\phi_{m}$ is bounded in $L^{\infty}\left(0, T ; H^{1}(\Omega)\right)$ together uniformely with $m$, we have

$$
\mathcal{B}_{m} \leq C\left\|c_{m}\right\|_{H^{1}(\Omega)}^{1 / 2}\left\|\nabla \phi_{m}\right\|_{H^{2}(\Omega)}^{1 / 2}\left\|\nabla \Delta \phi_{m}\right\|_{L^{2}(\Omega)} .
$$

Using Young's inequality, we deduce that for all $\delta>0$ :

$$
\mathcal{B}_{m} \leq C\left(\frac{\delta}{2}\left\|\nabla \Delta \phi_{m}\right\|_{L^{2}(\Omega)}^{2}+\frac{1}{2 \delta}\left(\left\|c_{m}\right\|_{H^{1}(\Omega)}\left\|\nabla \phi_{m}\right\|_{H^{2}(\Omega)}\right)\right) .
$$

Moreover, the elliptic estimate (8) gives

$$
\left\|\phi_{m}\right\|_{H^{3}(\Omega)} \leq C\left(\left\|\Delta \phi_{m}\right\|_{H^{1}(\Omega)}+\left\|\phi_{m}\right\|_{H^{1}(\Omega)}\right)
$$

and consequently

$$
\left\|\nabla \phi_{m}\right\|_{H^{2}(\Omega)} \leq C\left(\left\|\Delta \phi_{m}\right\|_{L^{2}(\Omega)}+\left\|\phi_{m}\right\|_{H^{1}(\Omega)}+\left\|\nabla \Delta \phi_{m}\right\|_{L^{2}(\Omega)}\right) .
$$

Using again Young's inequality, we have for all $\gamma>0$ :

$\mathcal{B}_{m} \leq C\left(\frac{\delta}{2}\left\|\nabla \Delta \phi_{m}\right\|_{L^{2}(\Omega)}^{2}+\frac{1}{2 \delta}\left(\frac{1}{2 \gamma}\left\|c_{m}\right\|_{H^{1}(\Omega)}^{2}+\frac{\gamma}{2}\left(\left\|\Delta \phi_{m}\right\|_{L^{2}(\Omega)}^{2}+\left\|\phi_{m}\right\|_{H^{1}(\Omega)}^{2}+\left\|\nabla \Delta \phi_{m}\right\|_{L^{2}(\Omega)}^{2}\right)\right)\right)$.

By choosing $\gamma=2 \delta^{2}$, we obtain

$$
\mathcal{B}_{m} \leq C\left(\delta\left\|\nabla \Delta \phi_{m}\right\|_{L^{2}(\Omega)}^{2}+\frac{1}{8 \delta^{3}}\left\|c_{m}\right\|_{H^{1}(\Omega)}^{2}+\frac{\delta}{2}\left(\left\|\Delta \phi_{m}\right\|_{L^{2}(\Omega)}^{2}+\left\|\phi_{m}\right\|_{H^{1}(\Omega)}^{2}\right)\right) .
$$

By choosing $C \delta=\frac{\varepsilon^{2}}{4}$, we obtain with $(47)$ :

$$
\mathcal{F}_{m} \leq \frac{\varepsilon^{2}}{2}\left\|\nabla \Delta \phi_{m}\right\|_{L^{2}(\Omega)}^{2}+C\left(\left\|c_{m}\right\|_{H^{1}(\Omega)}^{2}+\left\|\Delta \phi_{m}\right\|_{L^{2}(\Omega)}^{2}+\left\|\phi_{m}\right\|_{H^{1}(\Omega)}^{2}\right),
$$

that gives, with the identity (46) :

$$
\frac{d}{d t}\left\|\Delta \phi_{m}\right\|_{L^{2}(\Omega)}^{2}+\varepsilon^{2}\left\|\nabla \Delta \phi_{m}\right\|_{L^{2}(\Omega)}^{2} \leq C\left(\left\|\Delta \phi_{m}\right\|_{L^{2}(\Omega)}^{2}+\left\|\phi_{m}\right\|_{H^{1}(\Omega)}^{2}+\left\|c_{m}\right\|_{H^{1}(\Omega)}^{2}\right) .
$$

Integrating inequality $(50)$ over $(0, t)$ for $t \in(0, T)$ and using (21) i.e. the fact that $\phi_{m}$ and $c_{m}$ are bounded in $L^{2}\left(0, T ; H^{1}(\Omega)\right)$ uniformely with $m$, we get

$$
\left\|\Delta \phi_{m}(t)\right\|_{L^{2}(\Omega)}^{2}+\varepsilon^{2} \int_{0}^{t}\left\|\nabla \Delta \phi_{m}\right\|_{L^{2}(\Omega)}^{2} \leq\left\|\Delta \phi_{0 m}\right\|_{L^{2}(\Omega)}^{2}+C\left(1+\int_{0}^{t}\left\|\Delta \phi_{m}(t)\right\|_{L^{2}(\Omega)}^{2}\right) .
$$


Moreover, since $\left(\phi_{0}-\phi_{0 m}, v\right)_{L^{2}(\Omega)}=0$ for all $v \in V_{m}$, we obtain through iterated integrations by parts

$$
\begin{aligned}
\left\|\Delta \phi_{0 m}\right\|_{L^{2}(\Omega)}^{2} & =\left(\phi_{0 m}, \Delta^{2} \phi_{0 m}\right)_{L^{2}(\Omega)}=\left(\phi_{0}, \Delta^{2} \phi_{0 m}\right)_{L^{2}(\Omega)} \\
& =\left(\Delta \phi_{0}, \Delta \phi_{0 m}\right)_{L^{2}(\Omega)}-\int_{\partial \Omega} \frac{\partial \phi_{0}}{\partial n} \Delta \phi_{0 m} d \sigma .
\end{aligned}
$$

But since we assume that $\frac{\partial \phi_{0}}{\partial n}=0$ on $\partial \Omega$, the boundary term in (52) vanishes and we conclude by the Cauchy-Schwarz inequality that

$$
\left\|\Delta \phi_{0 m}\right\|_{L^{2}(\Omega)} \leq\left\|\Delta \phi_{0}\right\|_{L^{2}(\Omega)}
$$

Then applying Gronwall's lemma to inequality (51) and taking into account (53), we obtain that there exists a constant $C>0$ independent of $m$ such that

$$
\begin{gathered}
\left\|\Delta \phi_{m}\right\|_{L^{\infty}\left(0, T ; L^{2}(\Omega)\right)} \leq C, \\
\left\|\nabla \Delta \phi_{m}\right\|_{L^{2}\left(Q_{T}\right)} \leq C .
\end{gathered}
$$

We conclude from the elliptic estimate (8) and the uniform estimates (22),(35), (36), (54), (55) that

$$
\begin{gathered}
\left\|\phi_{m}\right\|_{L^{\infty}\left(0, T ; H^{2}(\Omega)\right)} \leq C, \\
\left\|\phi_{m}\right\|_{L^{2}\left(0, T ; H^{3}(\Omega)\right)} \leq C,
\end{gathered}
$$

where $C>0$ does not depend on $m$.

ii) Now, we choose $v=-\frac{\partial \Delta \phi_{m}}{\partial t} \in V_{m}$ in equation (12). Since $F_{1}, F_{2} \in W^{1, \infty}(\mathbb{R})$, we integrate by parts the resulting equation and owing to the homogeneous Neumann boundary condition satisfied by $\frac{\partial \phi_{m}}{\partial t}$ we obtain, as in the previous a priori estimate i) :

$$
\begin{aligned}
\left\|\nabla \frac{\partial \phi_{m}}{\partial t}\right\|_{L^{2}(\Omega)}^{2}+\frac{\varepsilon^{2}}{2} \frac{d}{d t}\left\|\Delta \phi_{m}\right\|_{L^{2}(\Omega)}^{2} \leq & \left(\left(\left\|\nabla \phi_{m}\right\|_{L^{2}(\Omega)}+\left\|\nabla c_{m}\right\|_{L^{2}(\Omega)}\right)\left\|\nabla \frac{\partial \phi_{m}}{\partial t}\right\|_{L^{2}(\Omega)}\right. \\
& \left.+\int_{\Omega}\left|c_{m} \nabla \phi_{m} \cdot \nabla \frac{\partial \phi_{m}}{\partial t}\right| d x\right) .
\end{aligned}
$$

We use Hölder's inequality to estimate the last term $\mathcal{C}_{m}=\int_{\Omega}\left|c_{m} \nabla \phi_{m} . \nabla \frac{\partial \phi_{m}}{\partial t}\right| d x$ in the right hand-side of (58) :

$$
\mathcal{C}_{m} \leq\left\|c_{m}\right\|_{L^{3}(\Omega)}\left\|\nabla \phi_{m}\right\|_{L^{6}(\Omega)}\left\|\nabla \frac{\partial \phi_{m}}{\partial t}\right\|_{L^{2}(\Omega)}
$$

Thanks to the embedding (41) and the uniform estimate (56) i.e. the fact that $\phi_{m}$ is bounded in $L^{\infty}\left(0, T ; H^{2}(\Omega)\right)$ uniformely with respect to $m$, we have

$$
\mathcal{C}_{m} \leq C\left(\left\|\nabla \frac{\partial \phi_{m}}{\partial t}\right\|_{L^{2}(\Omega)}\left\|c_{m}\right\|_{H^{1}(\Omega)}\right)
$$

Combining estimates (58) with (59) and using Young's inequality, we obtain

$$
\left\|\nabla \frac{\partial \phi_{m}}{\partial t}\right\|_{L^{2}(\Omega)}^{2}+\varepsilon^{2} \frac{d}{d t}\left\|\Delta \phi_{m}\right\|_{L^{2}(\Omega)}^{2} \leq C\left(\left\|\phi_{m}\right\|_{H^{1}(\Omega)}^{2}+\left\|c_{m}\right\|_{H^{1}(\Omega)}^{2}\right) .
$$


Integration of (60) from 0 to $T$ with the use of the uniform estimates (21) and (53) yields

$$
\left\|\nabla \frac{\partial \phi_{m}}{\partial t}\right\|_{L^{2}\left(Q_{T}\right)} \leq C
$$

and due to (35), we conclude that

$$
\left\|\frac{\partial \phi_{m}}{\partial t}\right\|_{L^{2}\left(0, T ; H^{1}(\Omega)\right.} \leq C
$$

where $C>0$ does not depend on $m$.

Additional a priori estimates for $c_{m}$.

iii) We choose $w=-\Delta c_{m} \in V_{m}$ in equation (13). Using the fact that from the assumptions (H2)-(H3) we have $D_{1} \in W^{1, \infty}(\mathbb{R})$ and $D_{2} \in W^{1, \infty}(\mathbb{R} \times \mathbb{R})$ and integrating by parts the equation resulting from this specific choice of the test function, we obtain

$$
\frac{1}{2} \frac{d}{d t}\left\|\nabla c_{m}\right\|_{L^{2}(\Omega)}^{2}+\int_{\Omega} \operatorname{div}\left(D_{1}\left(\phi_{m}\right) \nabla c_{m}\right) \Delta c_{m}=-\int_{\Omega} \operatorname{div}\left(D_{2}\left(c_{m}, \phi_{m}\right) \nabla \phi_{m}\right) \Delta c_{m} .
$$

Hence, using assumption (H2)-(H3) we get

$$
\begin{aligned}
\frac{1}{2} \frac{d}{d t}\left\|\nabla c_{m}\right\|_{L^{2}(\Omega)}^{2}+D_{s}\left\|\Delta c_{m}\right\|_{L^{2}(\Omega)}^{2} \leq C & \left(\int_{\Omega}\left|\nabla \phi_{m} \cdot \nabla c_{m} \Delta c_{m}\right|+\int_{\Omega}\left|\nabla \phi_{m}\right|^{2}\left|\Delta c_{m}\right|\right. \\
& \left.+\int_{\Omega}\left|\Delta \phi_{m} \Delta c_{m}\right|\right) .
\end{aligned}
$$

We set $\mathcal{E}_{1}=\int_{\Omega}\left|\nabla \phi_{m} \cdot \nabla c_{m} \Delta c_{m}\right|, \quad \mathcal{E}_{2}=\int_{\Omega}\left|\nabla \phi_{m}\right|^{2}\left|\Delta c_{m}\right| \quad$ and $\quad \mathcal{E}_{3}=\int_{\Omega}\left|\Delta \phi_{m} \Delta c_{m}\right|$. By Hölder's and Young's inequalities and thanks to the embedding (41) and the uniform estimate (56) i.e. the fact that $\phi_{m}$ is bounded in $L^{\infty}\left(0, T ; H^{2}(\Omega)\right)$ uniformely with respect to $m$, we deduce that

$$
\begin{aligned}
\mathcal{E}_{2} & \leq C\left\|\nabla \phi_{m}\right\|_{L^{4}(\Omega)}^{2}\left\|\Delta c_{m}\right\|_{L^{2}(\Omega)} \leq \frac{D_{s}}{6}\left\|\Delta c_{m}\right\|_{L^{2}(\Omega)}^{2}+C\left\|\phi_{m}\right\|_{L^{\infty}\left(0, T ; H^{2}(\Omega)\right)}^{4} \\
& \leq \frac{D_{s}}{6}\left\|\Delta c_{m}\right\|_{L^{2}(\Omega)}^{2}+C
\end{aligned}
$$

and likewise

$$
\mathcal{E}_{3} \leq \frac{D_{s}}{6}\left\|\Delta c_{m}\right\|_{L^{2}(\Omega)}^{2}+C .
$$

To estimate $\mathcal{E}_{1}$ we proceed as follows. We use Hölder's inequality and the embedding (41) with (56) i.e. the fact that $\phi_{m}$ is bounded $L^{\infty}\left(0, T ; H^{2}(\Omega)\right)$ uniformely with $m$ and then the Gagliardo-Nirenberg inequality (42) in order to get, if $C_{1}, C_{2}, \ldots$ are constants independent of $m$ :

$$
\begin{aligned}
\mathcal{E}_{1} & \leq C_{1}\left\|\nabla \phi_{m}\right\|_{L^{6}(\Omega)}\left\|\nabla c_{m}\right\|_{L^{3}(\Omega)}\left\|\Delta c_{m}\right\|_{L^{2}(\Omega)} \leq C_{2}\left\|\phi_{m}\right\|_{H^{2}(\Omega)}\left\|\nabla c_{m}\right\|_{L^{3}(\Omega)}\left\|\Delta c_{m}\right\|_{L^{2}(\Omega)} \\
& \leq C_{3}\left\|\nabla c_{m}\right\|_{H^{1}(\Omega)}^{1 / 2}\left\|\nabla c_{m}\right\|_{L^{2}(\Omega)}^{1 / 2}\left\|\Delta c_{m}\right\|_{L^{2}(\Omega)} \leq C_{4}\left\|c_{m}\right\|_{H^{2}(\Omega)}^{1 / 2}\left\|\nabla c_{m}\right\|_{L^{2}(\Omega)}^{1 / 2}\left\|\Delta c_{m}\right\|_{L^{2}(\Omega)} .
\end{aligned}
$$

Now, by Young's inequality and the elliptic estimate (8) we obtain

$$
\begin{aligned}
\mathcal{E}_{1} & \leq \frac{D_{s}}{12}\left\|\Delta c_{m}\right\|_{L^{2}(\Omega)}^{2}+C_{5}\left(\left\|\Delta c_{m}\right\|_{L^{2}(\Omega)}+\left\|c_{m}\right\|_{L^{2}(\Omega)}\right)\left\|\nabla c_{m}\right\|_{L^{2}(\Omega)} \\
& \leq \frac{D_{s}}{6}\left\|\Delta c_{m}\right\|_{L^{2}(\Omega)}^{2}+C_{6}\left(\left\|c_{m}\right\|_{L^{2}(\Omega)}^{2}+\left\|\nabla c_{m}\right\|_{L^{2}(\Omega)}^{2}\right) \\
& \leq \frac{D_{s}}{6}\left\|\Delta c_{m}\right\|_{L^{2}(\Omega)}^{2}+C_{7}\left(1+\left\|\nabla c_{m}\right\|_{L^{2}(\Omega)}^{2}\right)
\end{aligned}
$$


thanks to (22). Hence, the estimates (63), (64) and (65) in (62) imply there exists $C$ such that

$$
\frac{d}{d t}\left\|\nabla c_{m}\right\|_{L^{2}(\Omega)}^{2}+D_{s}\left\|\Delta c_{m}\right\|_{L^{2}(\Omega)}^{2} \leq C\left(1+\left\|\nabla c_{m}\right\|_{L^{2}(\Omega)}^{2}\right)
$$

Since we suppose $c_{0} \in H^{1}(\Omega)$, we infer from (11) that $\left\|\nabla c_{0 m}\right\|_{L^{2}(\Omega)} \leq\left\|\nabla c_{0}\right\|_{L^{2}(\Omega)}$. Thus applying Gronwall's lemma to (66) and then using the elliptic estimate (8) with (21) we conclude that there exists a constant $C>0$ independent of $m$ such that

$$
\begin{aligned}
\left\|c_{m}\right\|_{L^{\infty}\left(0, T ; H^{1}(\Omega)\right)} & \leq C, \\
\left\|c_{m}\right\|_{L^{2}\left(0, T ; H^{2}(\Omega)\right)} & \leq C .
\end{aligned}
$$

iv) We choose $w=\frac{\partial c_{m}}{\partial t} \in V_{m}$ in equation (13). Using the fact that $D_{1} \in W^{1, \infty}(\mathbb{R})$ and $D_{2} \in W^{1, \infty}(\mathbb{R} \times \mathbb{R})$ and integrating by parts we obtain

$$
\left\|\frac{\partial c_{m}}{\partial t}\right\|_{L^{2}(\Omega)}^{2}=\int_{\Omega} \operatorname{div}\left(D_{1}\left(\phi_{m}\right) \nabla c_{m}+D_{2}\left(c_{m}, \phi_{m}\right) \nabla \phi_{m}\right) \frac{\partial c_{m}}{\partial t} .
$$

Hence, using assumption (H2)-(H3) we get

$$
\left\|\frac{\partial c_{m}}{\partial t}\right\|_{L^{2}(\Omega)}^{2} \leq C \int_{\Omega}\left(\left|\nabla \phi_{m} . \nabla c_{m}\right|+\left|\nabla \phi_{m}\right|^{2}+\left|\Delta \phi_{m}\right|+\left|\Delta c_{m}\right|\right)\left|\frac{\partial c_{m}}{\partial t}\right| .
$$

We proceed as in (62) to estimate the right hand-side of (69). This leads to

$$
\left\|\frac{\partial c_{m}}{\partial t}\right\|_{L^{2}(\Omega)}^{2} \leq C\left(1+\left\|c_{m}\right\|_{H^{2}(\Omega)}^{2}\right)
$$

Hence, according to (68), we have that

$$
\left\|\frac{\partial c_{m}}{\partial t}\right\|_{L^{2}\left(Q_{T}\right)} \leq C
$$

where $C>0$ does not depend on $m$.

Now we are able to establish the regularity result. Indeed, we deduce from (56), (57) and (61) that

$$
\phi \in L^{2}\left(0, T ; H^{3}(\Omega)\right) \cap H^{1}\left(0, T ; H^{1}(\Omega)\right) .
$$

Moreover, thanks to (68) and (70), $c_{m}$ is bounded in the space $W_{3}$ defined by (38) and which is compactly embedded into $L^{2}\left(0, T ; H^{1}(\Omega)\right)$. Then

$$
c \in L^{2}\left(0, T ; H^{2}(\Omega)\right) \cap H^{1}\left(0, T ; L^{2}(\Omega)\right)
$$

and there exists a subsequence still denoted by $c_{m}$ such that as $m \rightarrow+\infty$,

$$
\begin{array}{ll}
c_{m} \rightarrow c & \text { in } L^{2}\left(0, T ; H^{1}(\Omega)\right) \text { strongly, } \\
\frac{\partial c_{m}}{\partial t} \rightarrow \frac{\partial c}{\partial t} & \text { in } L^{2}\left(Q_{T}\right) \text { weakly. }
\end{array}
$$

We then pass to the limit in Problem $\left(P_{m}\right)$ and find that $c$ and $\phi$ satisfy (45). 
b) Uniqueness. Suppose there exist two solutions $\left(\phi_{1}, c_{1}\right)$ and $\left(\phi_{2}, c_{2}\right)$ satisfying (44) and (45) together with the same initial data. We note $\phi=\phi_{2}-\phi_{1}$ and $c=c_{2}-c_{1}$ and derive some estimates for $\phi$ and $c$. In the sequel, we will denote by $C$ a positive constant depending on $\varepsilon^{2}, M_{1}, M_{2}, D_{s}, D_{l}, T$, on the Lipschitz constants of nonlinear terms $F_{i}, D_{i}, i=1,2$ and also on $\left\|\phi_{2}\right\|_{L^{\infty}\left(0, T ; H^{1}(\Omega)\right)}$ and $\left\|c_{2}\right\|_{L^{\infty}\left(0, T ; H^{1}(\Omega)\right)}$.

i) First, we multiply the equation for $\phi$ by $\phi$ and integrate over $\Omega$. We obtain after integrating by parts, for a.e. $t \in(0, T)$

$$
\begin{aligned}
\frac{1}{2} \frac{d}{d t}\|\phi(t)\|_{L^{2}(\Omega)}^{2}+\varepsilon^{2}\|\nabla \phi(t)\|_{L^{2}(\Omega)}^{2} & =\int_{\Omega}\left(F_{1}\left(\phi_{2}\right)-F_{1}\left(\phi_{1}\right)\right) \phi d x+\int_{\Omega} F_{2}\left(\phi_{1}\right) c \phi d x \\
& +\int_{\Omega} c_{2}\left(F_{2}\left(\phi_{2}\right)-F_{2}\left(\phi_{1}\right)\right) \phi d x .
\end{aligned}
$$

Using assumption (H1) with the Hölder and the Cauchy-Schwarz inequalities, we get

$$
\begin{aligned}
\frac{1}{2} \frac{d}{d t}\|\phi(t)\|_{L^{2}(\Omega)}^{2}+\varepsilon^{2}\|\nabla \phi(t)\|_{L^{2}(\Omega)}^{2} \leq C & \left(\|\phi(t)\|_{L^{2}(\Omega)}^{2}+\|c(t)\|_{L^{2}(\Omega)}^{2}\right. \\
& \left.+\left\|c_{2}(t)\right\|_{L^{4}(\Omega)}\|\phi(t)\|_{L^{4}(\Omega)}\|\phi(t)\|_{L^{2}(\Omega)}\right) .
\end{aligned}
$$

Thanks to the continuous embedding $H^{1}(\Omega) \hookrightarrow L^{4}(\Omega)$ and since $c_{2} \in L^{\infty}\left(0, T ; H^{1}(\Omega)\right)$, we obtain

$$
\frac{1}{2} \frac{d}{d t}\|\phi(t)\|_{L^{2}(\Omega)}^{2}+\varepsilon^{2}\|\nabla \phi(t)\|_{L^{2}(\Omega)}^{2} \leq C\left(\|\phi(t)\|_{L^{2}(\Omega)}^{2}+\|c(t)\|_{L^{2}(\Omega)}^{2}+\|\nabla \phi(t)\|_{L^{2}(\Omega)}\|\phi(t)\|_{L^{2}(\Omega)}\right) .
$$

We conclude by Young's inequality that

$$
\frac{d}{d t}\|\phi(t)\|_{L^{2}(\Omega)}^{2}+\varepsilon^{2}\|\nabla \phi(t)\|_{L^{2}(\Omega)}^{2} \leq C\left(\|\phi(t)\|_{L^{2}(\Omega)}^{2}+\|c(t)\|_{L^{2}(\Omega)}^{2}\right) .
$$

ii) We multiply the equation for $\phi$ by $-\Delta \phi$ and integrate over $\Omega$. We obtain after integrating by parts, for a.e. $t \in(0, T)$ :

$$
\begin{aligned}
\frac{1}{2} \frac{d}{d t}\|\nabla \phi(t)\|_{L^{2}(\Omega)}^{2}+\varepsilon^{2}\|\Delta \phi(t)\|_{L^{2}(\Omega)}^{2} & =-\int_{\Omega}\left(F_{1}\left(\phi_{2}\right)-F_{1}\left(\phi_{1}\right)\right) \Delta \phi d x-\int_{\Omega} F_{2}\left(\phi_{1}\right) c \Delta \phi d x \\
& -\int_{\Omega} c_{2}\left(F_{2}\left(\phi_{2}\right)-F_{2}\left(\phi_{1}\right)\right) \Delta \phi d x .
\end{aligned}
$$

Using assumption (H1) and Hölder's inequality, we get

$$
\begin{aligned}
\frac{1}{2} \frac{d}{d t}\|\nabla \phi(t)\|_{L^{2}(\Omega)}^{2}+\varepsilon^{2}\|\Delta \phi(t)\|_{L^{2}(\Omega)}^{2} \leq C & \left(\|\phi(t)\|_{L^{2}(\Omega)}+\|c(t)\|_{L^{2}(\Omega)}\right. \\
& \left.+\left\|c_{2}(t)\right\|_{L^{4}(\Omega)}\|\phi(t)\|_{L^{4}(\Omega)}\right)\|\Delta \phi(t)\|_{L^{2}(\Omega)} .
\end{aligned}
$$

We conclude as in the previous part i) that

$$
\frac{d}{d t}\|\nabla \phi(t)\|_{L^{2}(\Omega)}^{2}+\varepsilon^{2}\|\Delta \phi(t)\|_{L^{2}(\Omega)}^{2} \leq C\left(\|\phi(t)\|_{L^{2}(\Omega)}^{2}+\|c(t)\|_{L^{2}(\Omega)}^{2}+\|\nabla \phi(t)\|_{L^{2}(\Omega)}^{2}\right) .
$$

iii) We multiply the equation for $c$ by $c$ and integrate over $\Omega$. We obtain after integrating by parts, for a.e. $t \in(0, T)$ :

$$
\begin{aligned}
\frac{1}{2} \frac{d}{d t}\|c(t)\|_{L^{2}(\Omega)}^{2} & +\int_{\Omega}\left(D_{1}\left(\phi_{2}\right) \nabla c_{2}-D_{1}\left(\phi_{1}\right) \nabla c_{1}\right) \cdot \nabla c d x \\
& +\int_{\Omega}\left(D_{2}\left(c_{2}, \phi_{2}\right) \nabla \phi_{2}-D_{2}\left(c_{1}, \phi_{1}\right) \nabla \phi_{1}\right) \cdot \nabla c d x=0
\end{aligned}
$$


that we write in the following form

$$
\begin{aligned}
\frac{1}{2} \frac{d}{d t}\|c(t)\|_{L^{2}(\Omega)}^{2}+\int_{\Omega} D_{1}\left(\phi_{1}\right)|\nabla c|^{2} d x & =\int_{\Omega}\left(D_{1}\left(\phi_{1}\right)-D_{1}\left(\phi_{2}\right)\right) \nabla c_{2} \cdot \nabla c d x-\int_{\Omega} D_{2}\left(c_{1}, \phi_{1}\right) \nabla \phi \cdot \nabla c d x \\
& +\int_{\Omega}\left(D_{2}\left(c_{1}, \phi_{1}\right)-D_{2}\left(c_{2}, \phi_{2}\right)\right) \nabla \phi_{2} \cdot \nabla c d x .
\end{aligned}
$$

Hence, using assumptions (H2) and (H3), we get

$$
\begin{aligned}
\frac{1}{2} \frac{d}{d t}\|c(t)\|_{L^{2}(\Omega)}^{2}+D_{s}\|\nabla c(t)\|_{L^{2}(\Omega)}^{2} \leq & C\left(\int_{\Omega}|\phi|\left|\nabla c_{2} \cdot \nabla c\right| d x+\int_{\Omega}|\nabla \phi \cdot \nabla c| d x\right. \\
& \left.+\int_{\Omega}(|\phi|+|c|)\left|\nabla \phi_{2} \cdot \nabla c\right| d x\right) .
\end{aligned}
$$

We have to evaluate the right hand-side of inequality (75). Using the continuous embedding $H^{2}(\Omega) \hookrightarrow L^{\infty}(\Omega)$ which is valid for a smooth domain $\Omega \subset \mathbb{R}^{d}$ with $d \leq 3$ and owing to the elliptic estimate (8), there exist positive constants $C_{1}, C_{2}$ such that

$$
\begin{aligned}
\int_{\Omega}|\phi|\left|\nabla c_{2} . \nabla c\right| d x & \leq\|\phi\|_{L^{\infty}(\Omega)}\left\|\nabla c_{2}\right\|_{L^{2}(\Omega)}\|\nabla c\|_{L^{2}(\Omega)} \\
& \leq C_{1}\left\|c_{2}\right\|_{L^{\infty}\left(0, T ; H^{1}(\Omega)\right)}\|\phi\|_{H^{2}(\Omega)}\|\nabla c\|_{L^{2}(\Omega)} \\
& \leq C_{2}\left(\|\Delta \phi\|_{L^{2}(\Omega)}+\|\phi\|_{L^{2}(\Omega)}\right)\|\nabla c\|_{L^{2}(\Omega)}
\end{aligned}
$$

The same estimate holds for the term $\int_{\Omega}|\phi|\left|\nabla \phi_{2} . \nabla c\right| d x$. Moreover, by the use of the Hölder inequality, the continuous embedding (41) and the Gagliardo-Nirenberg inequality (42), there exist positive constants $C_{3}, C_{4}$ such that

$$
\begin{aligned}
\int_{\Omega}|c|\left|\nabla \phi_{2} \cdot \nabla c\right| d x & \leq\|c\|_{L^{3}(\Omega)}\left\|\nabla \phi_{2}\right\|_{L^{6}(\Omega)}\|\nabla c\|_{L^{2}(\Omega)} \\
& \leq C_{3}\left\|\phi_{2}\right\|_{L^{\infty}\left(0, T ; H^{2}(\Omega)\right)}\|c\|_{H^{1}(\Omega)}^{1 / 2}\|c\|_{L^{2}(\Omega)}^{1 / 2}\|\nabla c\|_{L^{2}(\Omega)} \\
& \leq C_{4}\left(\|c\|_{L^{2}(\Omega)}\|\nabla c\|_{L^{2}(\Omega)}+\|c\|_{L^{2}(\Omega)}^{1 / 2}\|\nabla c\|_{L^{2}(\Omega)}^{3 / 2}\right) .
\end{aligned}
$$

Then, from the estimates (76) and (77) in (75) with the use of Young's inequality, we deduce there exists a positive constant $C$ such that for a.e. $t \in(0, T)$ :

$$
\frac{d}{d t}\|c(t)\|_{L^{2}(\Omega)}^{2}+D_{s}\|\nabla c(t)\|_{L^{2}(\Omega)}^{2} \leq C\left(\|\Delta \phi(t)\|_{L^{2}(\Omega)}^{2}+\|\phi(t)\|_{H^{1}(\Omega)}^{2}+\|c(t)\|_{L^{2}(\Omega)}^{2}\right) .
$$

Now, we multiply inequality (78) by $\mu>0$ to be choosen later and we add the resulting inequality with (73) and (74). This leads to

$$
\begin{aligned}
\frac{d}{d t}\left(\|\phi(t)\|_{H^{1}(\Omega)}^{2}+\mu\|c(t)\|_{L^{2}(\Omega)}^{2}\right)+\varepsilon^{2}\|\Delta \phi(t)\|_{L^{2}(\Omega)}^{2} & \leq\left(C_{5}+\mu C_{6}\right)\left(\|\phi(t)\|_{H^{1}(\Omega)}^{2}+\|c(t)\|_{L^{2}(\Omega)}^{2}\right) \\
& +\mu C_{6}\|\Delta \phi(t)\|_{L^{2}(\Omega)}^{2},
\end{aligned}
$$

where $C_{5}$ and $C_{6}$ are positive constants. Then we choose $\mu=\frac{\varepsilon^{2}}{C_{6}}>0$ in order to obtain

$$
\frac{d}{d t}\left(\|\phi(t)\|_{H^{1}(\Omega)}^{2}+\|c(t)\|_{L^{2}(\Omega)}^{2}\right) \leq C\left(\|\phi(t)\|_{H^{1}(\Omega)}^{2}+\|c(t)\|_{L^{2}(\Omega)}^{2}\right),
$$

where $C>0$ and for a.e. $t \in(0, T)$. Applying Gronwall's lemma and since $\phi(0)=c(0)=0$, we conclude that $\phi=c=0$ a.e. in $Q_{T}$. The uniqueness is then proved. 


\section{A maximum principle}

We establish a maximum principle under extra assumptions on the nonlinear terms. This result will allow us to conclude to the existence of solution to problem $(P)$ with the physical assumptions $(H)$. So, in addition to (H1), (H2) and (H3), we suppose that the nonlinear terms $F_{1}, F_{2}$, and $D_{2}$ satisfy the following extra assumptions:

(H4) $\cdot F_{1} \equiv F_{2} \equiv 0$ in $\left.]-\infty, 0\right] \cup[1,+\infty[$.

(H5) $\cdot D_{2}\left(\cdot, r_{2}\right) \equiv 0$ in $\left.]-\infty, 0\right] \cup\left[1,+\infty\left[\right.\right.$ and for all $r_{2} \in \mathbb{R}$.

Then we have the following result

Theorem 3 Let assumptions (H1)-(H5) be fulfilled.

Suppose that the initial data $\left(\phi_{0}, c_{0}\right) \in L^{2}(\Omega) \times L^{2}(\Omega)$ is such that

$$
0 \leq \phi_{0}(x), c_{0}(x) \leq 1 \text { for a.e. } x \in \Omega \text {. }
$$

Then for any $T>0$, every weak solution $(\phi, c) \in\left(L^{2}\left(0, T ; H^{1}(\Omega)\right)\right)^{2} \cap\left(H^{1}\left(0, T ; V^{\prime}\right)\right)^{2}$ satisfies for all $t \in[0, T]$

$$
0 \leq \phi(x, t), c(x, t) \leq 1 \text { for a.e. } x \in Q_{T} .
$$

Proof: We prove that if $\phi_{0}, c_{0} \geq 0$ a.e. in $\Omega$ then $\phi(t), c(t) \geq 0$ for all $t \in[0, T]$ and a.e. in $Q_{T}$. Let us consider the negative parts of $\phi$ and $c$ namely $\phi^{-}=\max (-\phi, 0)$ and $c^{-}=\max (-c, 0)$. According to [4], we have that $\phi^{-} \in L^{2}\left(0, T ; H^{1}(\Omega)\right)$ with $\nabla \phi^{-}=-\nabla \phi$ if $\phi<0$ and $\nabla \phi^{-}=0$ otherwise, a.e. in $Q_{T}$ and the same property holds for $c^{-}$. Then, we choose $v=-\phi^{-}$and $w=-c^{-}$in equations (9) to obtain for a.e. $t \in(0, T)$ :

$$
-<\frac{\partial \phi}{\partial t}, \phi^{-}>_{V^{\prime}, V}+\varepsilon^{2} \int_{\Omega}\left|\nabla \phi^{-}(t)\right|^{2} d x=-\int_{\Omega}\left(F_{1}(\phi)+c F_{2}(\phi)\right) \phi^{-} d x
$$

and

$$
-<\frac{\partial c}{\partial t}, c^{-}>_{V^{\prime}, V}+\int_{\Omega} D_{1}(\phi(t))\left|\nabla c^{-}(t)\right|^{2} d x=\int_{c<0} D_{2}(c, \phi) \nabla \phi . \nabla c d x .
$$

When a function $\psi$ belongs to $H^{1}\left(0, T ; H^{1}(\Omega)\right)$, according to [4] it is easy to prove that $<\frac{\partial \psi}{\partial t}, \psi^{-}>_{V^{\prime}, V}=-\frac{1}{2} \frac{d}{d t}\left\|\psi^{-}\right\|_{L^{2}(\Omega)}^{2}$ in $L^{1}(0, T)$. By using a density argument, it is possible to show that this equality holds for all functions $\psi$ in $L^{2}\left(0, T ; H^{1}(\Omega)\right) \cap H^{1}\left(0, T ; V^{\prime}\right)$. It follows that

$$
\frac{1}{2} \frac{d}{d t}\left\|\phi^{-}(t)\right\|_{L^{2}(\Omega)}^{2}+\varepsilon^{2} \int_{\Omega}\left|\nabla \phi^{-}(t)\right|^{2} d x=-\int_{\Omega}\left(F_{1}(\phi)+c F_{2}(\phi)\right) \phi^{-} d x
$$

and

$$
\frac{1}{2} \frac{d}{d t}\left\|c^{-}(t)\right\|_{L^{2}(\Omega)}^{2}+\int_{\Omega} D_{1}(\phi(t))\left|\nabla c^{-}(t)\right|^{2} d x=\int_{c<0} D_{2}(c, \phi) \nabla \phi \cdot \nabla c d x .
$$

Now, using assumption (H4) on the nonlinear terms $F_{1}$ and $F_{2}$, we see that

$$
\left(F_{1}(\phi)+c F_{2}(\phi)\right) \phi^{-}=0 \text { a.e. in } Q_{T} .
$$

On the other hand, assumption (H5) on $D_{2}$ leads to

$$
D_{2}(c, \phi)=0 \quad \text { if } c<0 \text {, a.e. in } Q_{T} \text {. }
$$


Thus, equations (80) and (81) are reduced to

$$
\frac{1}{2} \frac{d}{d t}\left\|\phi^{-}(t)\right\|_{L^{2}(\Omega)}^{2}+\varepsilon^{2} \int_{\Omega}\left|\nabla \phi^{-}(t)\right|^{2} d x=0
$$

and

$$
\frac{1}{2} \frac{d}{d t}\left\|c^{-}(t)\right\|_{L^{2}(\Omega)}^{2}+\int_{\Omega} D_{1}(\phi(t))\left|\nabla c^{-}(t)\right|^{2} d x=0
$$

for a.e. $t \in(0, T)$. Since $\phi_{0}^{-}=c_{0}^{-}=0$ and due to the fact that the function $D_{1}$ is positive, we conclude that $\phi^{-}(t)=c^{-}(t)=0$ for all $t \in[0, T]$ and a.e. in $\Omega$. If we want to prove that $\phi_{0}, c_{0} \leq 1$ a.e. in $\Omega$ imply $\phi(t), c(t) \leq 1$ for all $t \in[0, T]$ and a.e. in $Q_{T}$, it is sufficient to take $v=(\phi-1)^{+}$and $w=(c-1)^{+}$in equations $(9)$.

\section{Conclusion}

We are now able to conclude to the well-posedness of Problem $(P)$ with the physical assumptions $(H)$ for the nonlinear functions $F_{i}, D_{i}, i=1,2$. Recall that as we point out in Section 2, the physical modelling leads to nonlinear terms $F_{1}, F_{2}, D_{1}$ and $D_{2}$ which are smooth functions and $F_{1}, F_{2}$ can be choosen as polynomial functions of 4 th degree in the interval $[0,1]$ and any functions outside the interval $[0,1]$. A natural idea is to take polynomial functions of degree 4 on all the real line. Hence we cannot directly apply the existence and regularity results obtained in Section 3 and Section 4. However, due to the vanishing properties in $(H)$ of the physical nonlinear terms, that is $F_{1}(\phi)=F_{2}(\phi)=0$ for $\phi=0$ and $\phi=1$ and $D_{2}(c,)=$.0 for $c=0$ and $c=1$, we may truncate $F_{1}, F_{2}$ to 0 beyond the set $\{0<\phi<1\}$ and truncate $D_{2}$ to 0 beyond the set $\{0<c<1\}$. In that way, we obtain nonlinear functions $\tilde{F}_{1}, \tilde{F}_{2}$ and $\tilde{D}_{2}$ satisfying the Lipschitz and boundeness assumptions (H1),(H2),(H3) and then we can apply existence and regularity results of Section 3 and Section 4 . Moreover, since $\tilde{F}_{1}, \tilde{F}_{2}$ and $\tilde{D}_{2}$ also satisfy the vanishing assumptions (H4) and (H5), the maximum principle of Section 5 holds and since the initial physical data $c_{0}$ and $\phi_{0}$ are values belonging to the interval $[0,1]$, we are sure that the solution $(c, \phi)$ remains included between 0 and 1 when the time $t$ goes up. It follows that $c$ and $\phi$ don't depend on the choice of the extension of functions $F_{1}, F_{2}$, $D_{2}$ outside the interval $[0,1]$ and consequently the physical problem $(P)$ is well-posed.

\section{References}

[1] Brochet D., Chen X., Hilhorst D., 'Finite dimensional exponential attractor for the phase-field model', Applicable Anal., 49, 197-212 (1993).

[2] Caginalp G., 'An analysis of a phase-field model for free boundary', Arch. Rational Mech. Anal., 92, 205-245 (1986).

[3] Elliott C.M., Zheng S., 'Global existence and stability of solutions to the phase-field equations', in Free Boundary Value Problems, K. H. Hoffmann, J. Sprekels, eds., Internat. Ser. Num. Math., vol. 95, Birkhäuser, Basel, 1990, pp. 46-58.

[4] Gilbarg D., Trudinger N.S., Elliptic partial differential equations of second order, Springer-Verlag, Berlin 1983.

[5] Henry D., Geometric theory of semilinear parabolic equations, LNM 840, Springer-Verlag, Berlin Heidelberg New-York, 1981. 
[6] Kessler D., Ph.D. thesis, EPFL, in preparation.

[7] Kessler D., Krüger O., Scheid J.F., 'Construction d'un modèle de champ de phase à température homogène pour la solidification d'alliage binaire.', internal report DMAEPFL, 1998.

[8] Laurençot Ph., 'Weak solutions to a phase-field model with non-constant thermal conductivity', Quart. Appl. Math., 4, 739-760 (1997).

[9] Lions J.L., Magenes E., Problèmes aux limites non homogènes et applications, vol. 1, Dunod, Paris, 1968.

[10] Simon J., 'Compact sets in the space $L^{p}(0, T ; B)$ ', Ann. Mat. Pura Appl., 146, 65-96 (1987).

[11] Temam R., Navier-Stokes equations, North-Holland, Amsterdam, 1984.

[12] Warren J.A., Boettinger W.J., 'Prediction of dendritic growth and microsegregation patterns in a binary alloy using the phase-field model', Acta metall. mater., 43 (2), 689-703 (1995). 